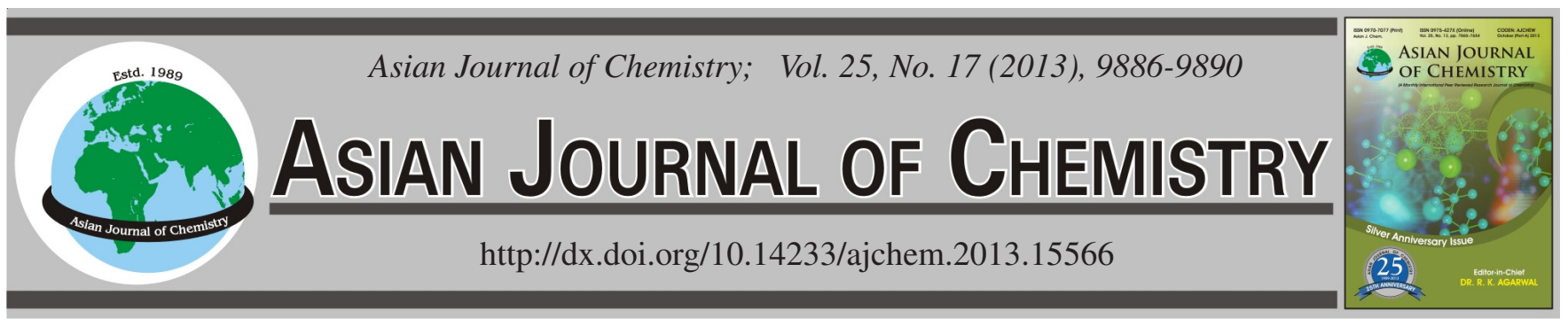

\title{
Biochemical and Pharmacological Study of Venom from Centipede Scoropendra subspinipes mutilans Koch
}

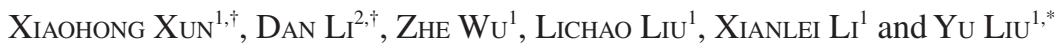

\begin{abstract}
${ }^{1}$ College of Chemistry and Chemical Engineering, Hunan Institute of Science and Technology, Yueyang 414006, P.R. China
\end{abstract} ${ }^{2}$ College of Life Science, Hunan Normal University, Changsha 410081, P.R. China

*Corresponding author: E-mail: hiliu2000@126.com

$\uparrow$ These authors contributed equally to this work.

\begin{abstract}
Almost all centipedes are predators and have venom glands. The primary purpose of centipede venoms is to kill or paralyze prey. Centipede venoms are complex chemical cocktails in which proteins are the main components. In this study, the biochemical and pharmacological properties of the venom of the centipede Scoropendra subspinipes mutilans Koch was investigated. The RP-HPLC and MALDI-TOF MS spectra of the venom indicated that it contains diverse peptides with molecular weights from 3000-10000 Da. Using the whole-cell patch-clamp, it was found that the venom could inhibit both voltage-gated sodium channels (VGSC) and potassium channels (VGPC) in adult rat dorsal root ganglion (DRG) neurons. Furthermore, pharmacological activities of the 43 fractions collected from RP-HPLC were examined on both VGSCs and VGPCs in rat DRG neurons. Consequently, we identified four fractions containing peptide neurotoxins which could inhibit VGSCs, as well as the other four fractions that containing peptide neurotoxins which could inhibit VGPCs. This study indicates that the venom of the centipede Scoropendra subspinipes mutilans Koch is indeed a rich source of novel pharmacologically interesting peptides that would receive increased attention from pharmacologists and biochemists in the future.
\end{abstract}

Key Words: Centipede venom, RP-HPLC, MALDI-TOF MS, Voltage-gated sodium channel, Voltage-gated potassium channel.

ᄂ $-\ldots-\ldots \ldots-\ldots-\ldots \ldots-\ldots-\ldots-\ldots-\ldots-\ldots$

\section{INTRODUCTION}

Almost 3,300 centipede species have been found in the world and most of them are equipped with venomous glands. Their first pair of walking legs is evolved into venomous appendages, also known as poison claws, forcipules, toxicognaths or maxillipeds ${ }^{1}$. They feed on a variety of vertebrate and invertebrate prey, including bats, rats, amphibians and reptiles ${ }^{1}$. They have developed complicated chemical strategies to capture prey and protect themselves from their own predators ${ }^{2,3}$. Centipede venoms cause instant paralysis in houseflies, cockroaches and crickets, which suggests that there are neurotoxins in centipede venoms that provide an efficient mean of rapidly paralyzing prey ${ }^{1,4,5}$. Besides, stings by several centipede species can inflict severe symptoms in humans, including myocardial ischemia and infarction ${ }^{6}$, hemorrhage ${ }^{7}$ and rhabdomyolysis ${ }^{8}$.

Although centipedes have long been known to be venomous but their venoms remain unexplored so far'. In the present study, we reported the biochemical and pharmacological properties of the venom from the centipede Scoropendra subspinipes mutilans Koch. This centipede, also known as
Chinese red-headed centipede, is a giant and venomous centipede found in East Asia and Australia. This centipede is found typically in damp, moist environments, where it grows to an average length of 8 inches. In China, there have been more than two thousand years for this centipede being a medicine, as recorded in Bencao Gangmu (also known as Compendium of Materia Medica), a famous medical book written 500 years ago. Here, we found that the venom contains various peptide toxins and some of them are able to inhibit voltage-gated sodium channels and potassium channels. Our study provides a clue for further identification of pharmacologically interesting peptides from the venom.

\section{EXPERIMENTAL}

Centipede and venom collection: The centipede Scoropendra subspinipes mutilans Koch were collected in Hubei province, China, maintained in plastic boxes that were covered with fallen leaves and given water daily. Chopped fishes were used to feed the animals. The venom was collected by electrostimulation ${ }^{10}$. About $100 \mathrm{mg}$ venom was pooled from approximately 500 centipedes, thus enabling exploration of the biochemical and pharmacological properties. The venom 
is clear and colorless liquid, easily soluble in water. After being freeze-dried, the venom was stored at $-20{ }^{\circ} \mathrm{C}$ prior to analysis.

RP-HPLC: The centipede venom was fractionated by reverse-phase high performance liquid chromatography (RPHPLC). Lyophilized venom, dissolved in double-distilled water, was applied onto a column (Vydac, C18. $300 \AA$, 4.6 $\mathrm{mm} \times 250 \mathrm{~mm}$ ) using Waters Alliance system. Venom components were eluted using a linear acetonitrile gradient (0-60\% acetonitrile $/ 0.1 \%$ TFA in $1 \mathrm{~h}$ ) at a flow rate of $1 \mathrm{~mL} / \mathrm{min}$. Elution of peptides was monitored at $215 \mathrm{~nm}$.

Mass spectrometry: The molecular masses of peptides were determined by using MALDI-TOF-TOF mass spectrometry (Ultra Flex I, Bruker Daltonics). Ionization was achieved by irradiation with nitrogen laser $(337 \mathrm{~nm})$, with a $20 \mathrm{kV}$ acceleration voltage. $\alpha$-Cyano-4-hydroxy-cimamic acid (CCA) was used as matrix.

Electrophysiological studies: Whole cell clamp recordings of voltage-gated sodium currents or potassium currents were made from rat DRG neurons, which were acutely dissociated from 30-day old Sprague-Dawley rats and maintained in short-term primary culture according to the methods described by Herzog ${ }^{11}$. Ionic currents were recorded from DRG neurons under whole-cell patch-clamp condition using an EPC-9 patchclamp amplifier (HEKA Electronics, Germany) at room temperature $\left(20-25^{\circ} \mathrm{C}\right)$. The patch pipettes with resistances of 2-3 $\mathrm{M} \Omega$ were fabricated from borosilicate glass tubing (VWR micropipettes, $100 \mu \mathrm{L}$, VWR company) using a two-stage vertical microelectrode puller (PC-10, Narishige, Japan) and fire-polished by a heater (Narishige, Japan). Voltage steps and data acquisition were controlled using a PC computer with software Pulsefit + Pulse 8.0 (HEKA Electronics, Germany). The P/4 protocol was used to subtract linear capacitive and leakage currents. The series resistance compensation was applied to $70-80 \%$ to minimize voltage errors. Voltage-gated sodium and potassium currents were recoded as the method described ${ }^{12,13}$.

\section{RESULTS AND DISCUSSION}

The venom from the centipede Scolopendra subspinipes mutilans was fractionated by RP-HPLC on a Vydac C18 column (Fig. 1A). Forty three fractions were detected and collected, indicating that the peptide toxins from the venom were diverse. MALDI-TOF mass spectrometry was further applied to determine the molecular masses of peptide toxins and elucidate the complexity of venomous peptides. Fig. 1B shows the molecular weight profile of the venom. It seems that the molecular weights are not very diverse as predicated. Less than 20 peaks would be detected on the mass spectrometry. We guessed that the diversity of the peptide toxins was underestimated. This might be resulted from the reasons as followed: (1) the centipede venom contains many salts and low molecular weight compounds, which would interfere with the ionization of peptide toxins; (2) some peptide toxins have very similar molecular weights, which could not be distinguished by MALDI-TOF mass spectrometry; (3) many peptide toxins are of very low amounts, which were not able to be detected. Actually, only several peaks showed high intensities in the mass spectrometry, while the intensities of most peaks are less than 500, which also indicated that only few peptides are of
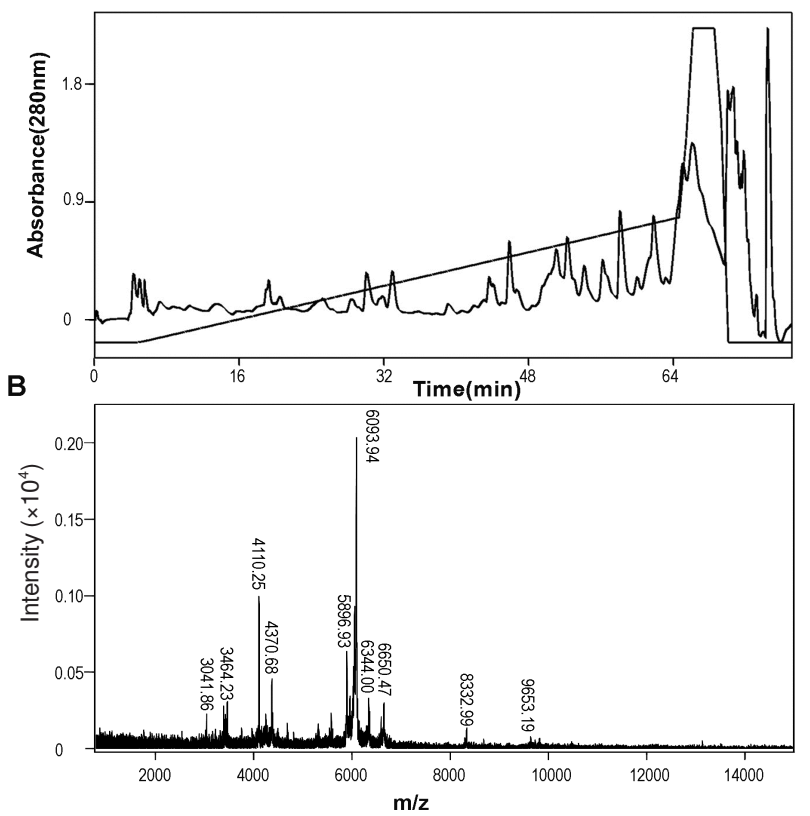

Fig. 1. RP-HPLC chromatography and MALDI-TOF MS spectrometry of the venom from the centipede Scoropendra subspinipes mutilans. (A) The venom was fractionated by RP-HPLC on a Vydac C18 column $(300 \AA$, $4.6 \mathrm{~mm} \times 250 \mathrm{~mm})$. The peptides were eluted using a linear gradient of $0-60 \%$ acetonitrile/ $0.1 \%$ TFA for $60 \mathrm{~min}$ at a flow rate of $1.0 \mathrm{~mL} / \mathrm{min}$. (B) MALDI-TOF MS analysis of the venom demonstrated the molecular mass distribution

high abundances, while the amounts of most peptides are very low in the venom. However, it would still be explained that the molecular mass distribution is in the range from 3,000 to $10,000 \mathrm{Da}$ and concentrates in the range from 3,000-6,000 $\mathrm{Da}$. This suggested that most of peptide toxins in the venom contain about 30-50 amino acid residues.

Furthermore, all the 43 fractions from RP-HPLC would be submitted to MALDI-TOF mass spectrometry analysis. The data was listed in Table-1. Here, we could find that most fractions were still composed of multiple components with different molecular masses, indicating the diversity and complexity of the peptide toxins from the venom. In addition, from the retention time of 0-39 min, 23 fractions in total were collected. Except the fraction with retention time of 28-30 min, the other 22 fractions were showing evident absorbance at $215 \mathrm{~nm}$ in RP-HPLC spectra, but they could not be detected in MALDI-TOF MS analysis. This might be due to the following two reasons: (1) these fractions mainly contain low molecular compounds whose molecular weights were less than $1,000 \mathrm{Da}$ and were filtered in MALDI-TOF mass spectrometry analysis; (2) peptides in these fractions were of too low amounts to be detected in MALDI-TOF mass spectrometry analysis. This also indicated that most of the peptides in the venom were rather hydrophobic since they were eluted with acetonitrie concentration higher than $35 \%$.

Electrophysiological assays: Previous studies showed that there are neurotoxins in centipede venoms. Actually, our study indicated that the venom from the centipede Scoropendra subspinipes mutilans Koch could inhibit VGSCs and VGPCs in DRG neurons. As shown in (Fig. 2), the venom was able to inhibit $32 \%$ sodium currents (Fig. 2A) and $17 \%$ potassium 
TABLE-1

SUMMARY OF MOLECULAR WEIGHTS AND ACTIVITIES OF FRACTIONS FROM RP-HPLC OF THE VENOM

\begin{tabular}{cclcc}
\hline Index & Retention time $(\mathrm{min})$ & \multicolumn{1}{c}{ Molecular weight (Da) } & Inhibition on VGSCs & Inhibition on VGPCs \\
\hline 18 & $28.0-30.0$ & $1426.8,1582.8,1311.9$ & + & - \\
24 & $39.0-40.8$ & $3725.3,2988.1,1864.2$ & - & - \\
25 & $40.8-43.3$ & $3146.6,2964.2,1857.5,1290.9,4151.2,6045.0$ & - & - \\
26 & $43.3-44.4$ & $2970,3134,6055,5543,3570$ & - & - \\
27 & $44.4-45.4$ & $3132.7,1567,3359$ & + & - \\
28 & $45.4-46.4$ & 3132.9 & + & - \\
29 & $46.4-49.2$ & $3133.4,3440,3768$ & - & - \\
30 & $49.2-50.0$ & $3769,3444,4290,5337,5730,1885$ & - & - \\
31 & $50.0-52.2$ & $3413.8,4292,5329,6106$ & - & - \\
32 & $52.2-54.0$ & $6095.9,6040,4371,3723$ & - & - \\
33 & $54.0-55.4$ & $6028.8,4371,5900,3501,3011,3284$ & - & + \\
34 & $55.4-56.4$ & $4115.4,5903,6046,3391$ & - & - \\
35 & $56.4-57.6$ & $4116.4,3857$ & - & - \\
36 & $57.6-60.0$ & $4118.9,3982,3861,6365,5949$ & - & - \\
37 & $60.0-61.0$ & $6371.2,3181,3865,4119,5977$ & - & - \\
38 & $61.0-63.4$ & $3864.2,5587,3605,6367$ & - & - \\
39 & $63.4-64.7$ & 6609.6 & + & - \\
40 & $64.7-65.5$ & - & - & - \\
42 & $65.5-68.6$ & - & - & - \\
43 & $68.6-70.6$ & 6685.5 & + & - \\
\hline
\end{tabular}

currents (Fig. 2 B) at a concentration of $1 \mathrm{mg} / \mathrm{mL}$, respectively. This showed that the venom should contain components with inhibitory activities on VGSCs or VGPCs. Therefore, we tried to screen bioactive components in all 43 fractions collected in RP-HPLC purification.
A

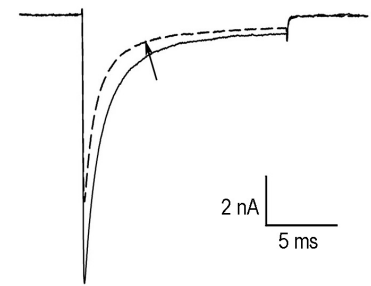

B

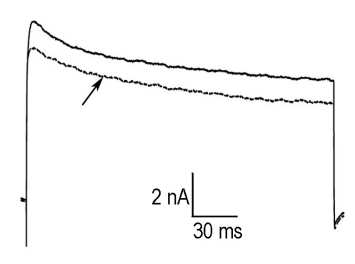

Fig. 2. Inhibitory activity of the venom on VGSCs (A) and VGPCs (B) in rat DRG neurons

We examined the potentially inhibitory activities of all the fractions on VGSCs in rat DRG neurons. It is well known that two types of VGSCs (TTX-S and TTX-R) existed in DRG neurons. TTX-S VGSCs activate and inactivate more quickly than TTX-R sodium currents. Both types of VGSCs can be distinguished by $\mathrm{TTX}^{14,15}$. As shown in Fig. 3, we found that four fractions exhibiting $\mathrm{s}$ inhibitory activity on VGSCs. Among them, two fractions were found to inhibit TTX-S VGSCs. The fraction, eluted in 28-30 min, contains a peptide with the molecular mass of 1428.06, indicating it has about 10 amino acid residues. This peptide was able to inhibit $70 \%$ of TTX-S sodium currents at a concentration of $100 \mathrm{nM}$ (Fig. 3A). The other fraction, eluted in 63.4-64.7 min, contains a peptide with the molecular mass of $6609.61 \mathrm{Da}$, and could inhibit $50 \%$ of sodium currents. Since the amount of this peptide was too low, the accurate concentration used in the experiment was not determined. The other two fractions displayed inhibitory effect on TTX-R VGSCs. They could reduce $32 \%$ and $21 \%$ of TTX-R sodium currents at concentrations

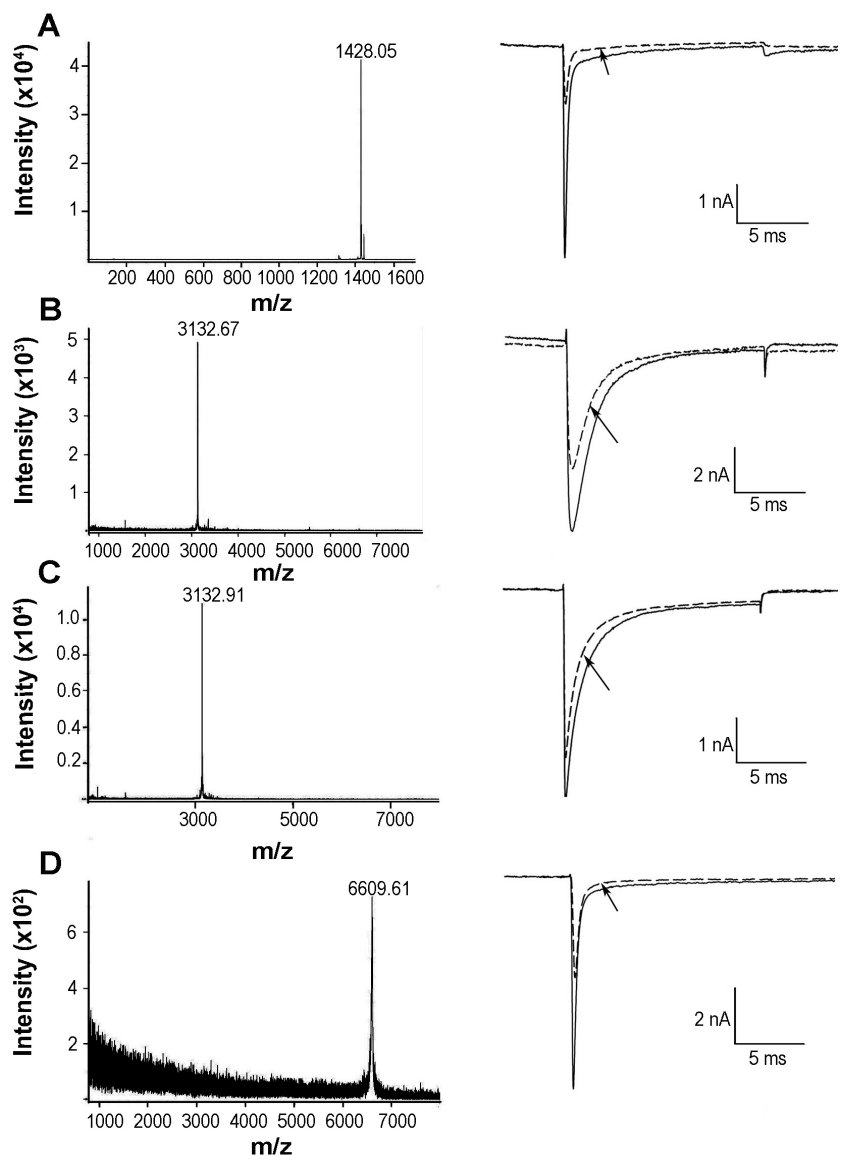

Fig. 3. Inhibitory acitvity of four fractions on VGSCs in rat DRG neurons. The four fractions were collected from the RP-HPLC purification of the venom. For each fraction, the molecular masses were determined by MALDI-TOF MS spectrometry (left panels), and the representative current traces recorded before and after the treatment with corresponding fraction were indicated (right panels). The current trace recorded after the treatment was labeled with an arrow. Note two fractions (A and D) could inhibit TTX-S VGSCs while the other two (C and D) could inhibit TTX-R VGSCs 
of $100 \mathrm{nM}$, respectively. They were eluted in 44.4-45.4 min and 45.4-46.4 min, respectively. It is interesting that both of them have the same molecular masses of about $3232 \mathrm{Da}$. We guessed that both peptides might share very high sequence identity.

We also identified four fractions that could inhibit VGPCs in rat DRG neurons. Dorsal root ganglion neurons exhibit two categories of potassium currents: transient and delayed rectifier outward potassium currents. As shown in Fig. 4, inhibition of four fractions on VGPCs are observed. The four fractions could be classified into two groups. One fraction, eluted in 70.6$73.2 \mathrm{~min}$, could inhibit $51 \%$ of transient outward potassium currents. Its molecular weight was determined as 6691.8 Da (Fig. 4D), indicating it is a peptide with about 60 amino acid residues. In addition, the amount of this peptide might be very low since its mass spectrometry displayed relatively low signal/ noise ratio. The other three fractions could inhibit delayed rectifier currents. They were eluted in three adjacent times: 55.4-56.4 $\mathrm{min}$, 56.4-57.6 min and 57.6-60.0 min, respectively. As revealed by MALDI-TOF MS spectrometry, the fraction with retention time of 55.4-56.4 min contained several
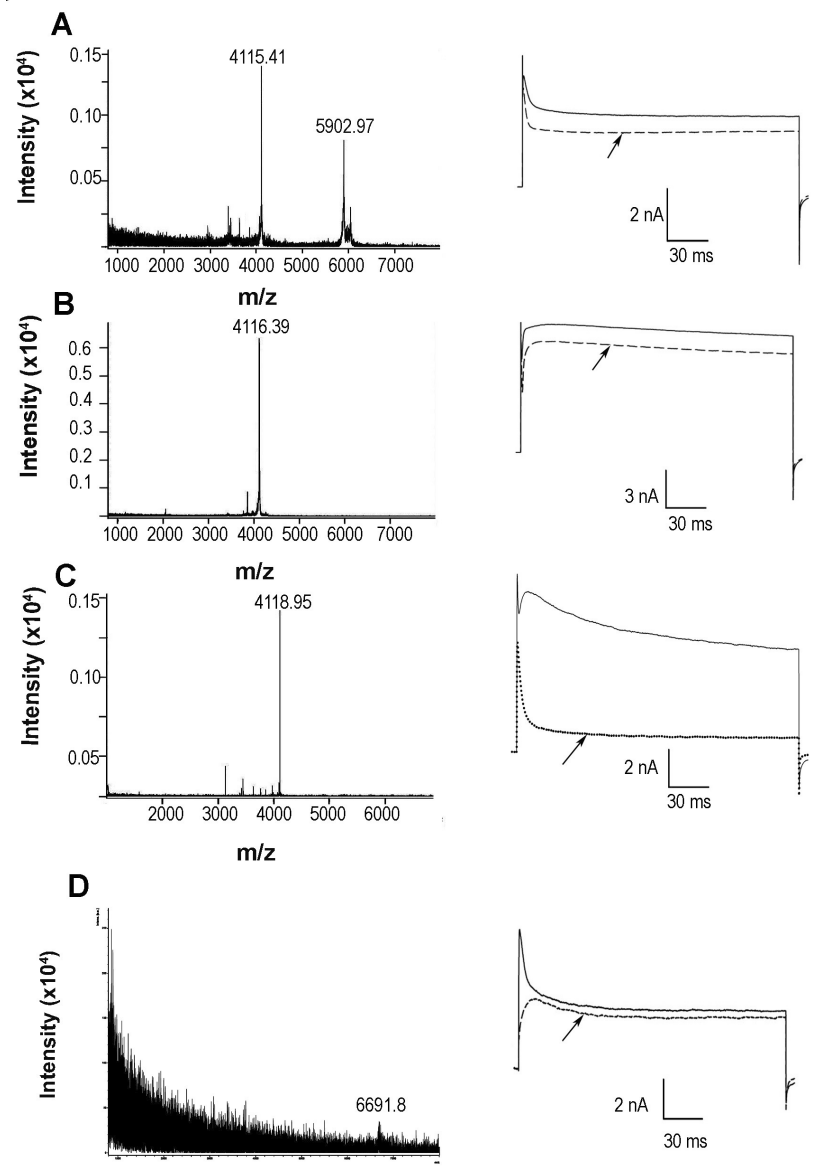

$\mathrm{m} / \mathrm{z}$

Fig. 4. Inhibitory acitvity of four fractions on VGPCs in rat DRG neurons. The four fractions were collected from the RP-HPLC purification of the venom. For each fraction, the molecular masses were determined by MALDI-TOF MS spectrometry (left panels), and the representative current traces recorded before and after the treatment with corresponding fraction were indicated (right panels). The current trace recorded after the treatment was labeled with an arrow. Note three fractions (A, B and C) could inhibit delayed rectification currents while the other one (D) could inhibit transient outward currents peptides, two of which might be the main components with molecular masses of 4115.41 Da and 5902.97 Da, respectively (Fig. 4A). Compared with this fraction, the purity of the following two fractions might be much higher. Their molecular masses were determined as 4116.39 Da and 4118.95 Da (Fig. 4B and 4C), respectively. Given the three peptides exhibit similar molecular masses, hydrophobicity and inhibitory activity, we assumed that they might share high sequence identity.

In the present study, we conducted a preliminary research of the biochemical and pharmacological properties of the venom from the centipede Scoropendra subspinipes mutilans. As revealed by RP-HPLC and MALDI-TOF MS spectra, the peptide toxins demonstrated a great diversity. The limited amount of the centipede venom could not allow us to yield sufficient peptide toxins with high purity. However, we still collected 43 fractions from the RP-HPLC chromatography and examined the inhibitory activity of each fraction on VGSCs and VGPCs in DRG neurons. Consequently, four fractions were found to antagonize VGSCs and another four fractions could inhibit VGPCs. Among the eight fractions, the purity of seven fractions is relative high as revealed by MALDI-TOF MS spectrometry. This allowed us to determine their amino acid sequences for further studies.

Voltage-gated sodium channels play important roles in initiating action potentials and nervous influx conduction in sensory nerves. They are the molecular targets for several types of neurotoxins that act at six or more distinct receptor sites ${ }^{16}$. Voltage-gated potassium channels are a diverse and ubiquitous family of membrane proteins expressed in both excitable and nonexcitable cells. They are involved in diverse physiological processes ranging from repolarization of neuronal or cardiac action potentials, over regulating calcium signaling and cell volume, to driving cellular proliferation and migration. Lots of evidences have shown that both types of ion channels are closely associated with many kinds of disorders. As a result, they have received much attention for their therapeutic potential. Voltage-gated sodium channels represent well-precedented drug targets as antidysrhythmics, anticonvulsants and local anaesthetics. Voltage-gated potassium channels offer tremendous opportunities for the development of new drugs for cancer, autoimmune diseases and metabolic, neurological and cardiovascular disorders. On the other hand, a large amount of researches have indicated that peptide toxins from animal venoms are capable of selectively acting on VGSCs or VGPCs. They have received increased attention as helpful probes for the investigation of structure and function of their targets, as well as idea leads for the development of therapeutic medicines treating related diseases. It is therefore widely accepted that animal venoms represent an important source for screening peptide toxins with good therapeutic efficacy.

In summary, we found that the venom from the centipede Scoropendra subspinipes mutilans contains diverse peptides and have identified antagonists of VGSCs and VGPCs. Significantly, these peptides displayed relative selectivity on interaction with VGSCs or VGPCs. For example, among the peptides acting on VGSCs, two preferentially inhibited TTX-S VGSCs, while the other two were apt to target on TTX-R VGSCs. So 
did the potassium channel toxins in the venom. They could also discriminate the two types of VGPCs in rat DRG neurouns. In this study, most fractions exhibit no or little effects on VGSCs and VGPCs in rat DRG neurons, but this might be due to their low amounts in the venom or alternatively because some of them had inhibitory effects on other ion channels instead of VGSCs and VGPCs. However, if sufficient venom could be collected, we still believed that more peptides would be identified as antagonists or modulators of VGSCs, VGPCs or other ion channels. This study would provide clues for explaining that centipede, as a generalist predator, uses venom to capture prey, as well as indicate that centipede venoms, like many venoms from many other animals, such as spiders, scorpions, snails, snakes, etc. are an interesting source of novel pharmaceuticals ${ }^{17}$ and may therefore have a role to play in venoms-based drug discovery programs.

\section{ACKNOWLEDGEMENTS}

This work was financially supported by the National Science Foundation of China (No. 21172067), the Science and Technology Department of Hunan Province (No. 2012SK3042) and the Science Foundation of Department of Education of Hunan Province (No. 13A032).

\section{REFERENCES}

1. E.A. Undheim and G.F. King, Toxicon, 57, 512 (2011)

2. B.G. Fry, K. Roelants, D.E. Champagne, H. Scheib, J. Tyndall, G.F.King, T.J. Nevalainen, J.A. Norman, R.J. Lewis, R.S. Norton, C. Renjifo and R.C. de la Vega, Annu. Rev. Genomics Hum. Genet., 10, 483 (2009).
3. B.G. Fry, N. Vidal, J.A. Norman, F.J. Vonk, H. Scheib, S.F. Ramjan, S.Kuruppu, K. Fung, S.B. Hedges, M.K. Richardson, W.C. Hodgson, V. Ignjatovic, R. Summerhayes and E.Kochva, Nature, 439, 584 (2006).

4. B. Rates, M.P. Bemquerer, M. Richardson, M.H. Borges, R.A. Morales, M.E. De Lima and A.M. Pimenta, Toxicon, 49, 810 (2007).

5. L. González-Morales, E. Diego-García, L. Segovia, C. Gutiérrez Mdel and L.D. Possani, Toxicon, 54, 8 (2009).

6. M. Ozsarac, O. Karcioglu, C. Ayrik, F. Somuncu and S. Gumrukcu, Wilderness, Environ. Med., 15, 109 (2004).

7. I. Kaysak, R. Martins and C.R. Bertim, Brazil. Rev. Saude Pub., 32, 514 (1998).

8. J.L. Logan and D.A. Ogden, West. J. Med., 142, 549 (1985).

9. H.W. Tedford, B.L. Sollod, F. Maggio and G.F. King, Toxicon, 43, 601 (2004).

10. K. Peng, Y. Kong, L. Zhai, X. Wu, P. Jia, J. Liu and H.Yu, Toxicon, 55, 274 (2010)

11. R.I. Herzog, T.R. Cummins, F. Ghassemi, S.D. Dib-Hajj and S.G. Waxman, J. Physiol., 551, 741 (2003).

12. J. Chen, Y. Zhang, M. Rong, L. Zhao, L. Jiang, D. Zhang, M. Wang, Y. Xiao and S. Liang, Peptides, 30, 1042 (2009).

13. D.L. Winkelman, C.L. Beck, D.L. Ypey and M.E. O'Leary, J. Pharmacol. Exp. Ther, 314, 1177 (2005).

14. B. Rates, M.P. Bemquerer, M. Richardson, M.H. Borges, R.A. Morales, M.E. De Lima and A.M. Pimenta, Toxicon, 49, 810 (2007).

15. S. Cestele and W.A. Catterall, Biochimie, 82, 883 (2000).

16. N. Ogata and Y. Ohishi, J. Pharmacol., 88, 365 (2002).

17. P. Escoubas and G.F. King, Expert. Rev. Proteomics, 6, 221 (2009). 